\title{
Du bon usage des corpus dans la recherche sur le discours spécifique
}

Jacky Martin

\section{(2) OpenEdition}

\section{Journals}

Édition électronique

URL : http://journals.openedition.org/asp/2984

DOI : 10.4000/asp.2984

ISSN : 2108-6354

\section{Éditeur}

Groupe d'étude et de recherche en anglais de spécialité

\section{Édition imprimée}

Date de publication : 1 décembre 1997

Pagination : 75-83

ISSN : 1246-8185

\section{Référence électronique}

Jacky Martin, «Du bon usage des corpus dans la recherche sur le discours spécifique », ASp [En ligne], 15-18 | 1997, mis en ligne le 03 mai 2002, consulté le 01 mai 2019. URL : http:// journals.openedition.org/asp/2984; DOI : 10.4000/asp.2984

Ce document a été généré automatiquement le 1 mai 2019.

Tous droits réservés 


\title{
Du bon usage des corpus dans la recherche sur le discours spécifique
}

\author{
Jacky Martin
}

1 Dans le cadre des études linguistiques, entendues au sens large, les corpus ont pris et prendront dorénavant une importance grandissante. On assiste à une multiplication des colloques ${ }^{1}$ et des numéros spéciaux de revues qui témoignent d'une phase intense de concertation et d'orientation dans la recherche. Déjà, les premiers produits issus directement de cet outil de recherche commencent à voir le jour. Des thèses, surtout en Grande-Bretagne, et des ouvrages accomplis comme ceux de M. Hoey (1991) ou J. Sinclair (1991) ou C. Gledhill (2000), parmi beaucoup d'autres, montrent que nous sommes en présence moins de l'amplification d'un phénomène déjà existant que d'une importante réorientation des études linguistiques. On est passé insensiblement d'une approche conceptualiste où seuls comptaient le modèle et l'illustration à un environnement de recherche ou les règles du jeu, tout en étant encore assez imprécises, paraissent radicalement différentes. La multiplication des corpus, leur taille phénoménale, l'exploitation systématique qui en est faite produisent des résultats à la fois nouveaux et dérangeants pour les grammaires traditionnelles. On voit s'affronter une linguistique du modèle, de la règle et de l'exemple avec une autre forme de linguistique qu'on pourrait qualifier, faute de qualificatif plus attesté, «de linguistique de corpus» pour laquelle seules comptent les régularités du corpus, les collocations et les combinaisons variables entre sémantique et syntaxe. Le modèle, s'il devait subsister, devient "à géométrie variable ", la règle est la somme de toutes les exceptions devenues indissociables des occurrences attestées, l'exemple, s'il est encore pertinent, fait le lien entre une gamme de possibles et de probables. Est-on en train d'assister à la naissance d'une nouvelle école linguistique, à un changement de paradigme, à la convergence de deux approches complémentaires ? Il est encore trop tôt pour le dire.

2 J'évoquerai certains de ces problèmes, au passage, mais ils dépassent de très loin mon propos immédiat qui est essentiellement pratique et limité à la sphère des études en langue spécifique. J'essaierai de voir dans quelle mesure cette ascension des corpus est 
résistible ou, pour dire les choses autrement, je montrerai de quelles précautions il faut s'entourer pour rendre leur utilisation et leur interprétation irrésistibles. Le problème de l'intégration des corpus dans le cadre des études sur le discours spécifique se pose en des termes un peu différents de celui de la linguistique des langues naturelles. Il se trouve que, depuis l'origine de ces études, le chercheur en langue spécifique a toujours eu recours, peu ou prou, aux corpus. La raison, fort simple, en était que son champ de recherche se confondait avec son champ d'exploration. Même s'il pouvait se prévaloir de divers savoirs, déjà fermement établis, tels que linguistique, terminologie, sociolinguistique, il lui fallait, d'abord et toujours, établir la spécificité de son objet d'étude. Cette spécificité, il ne pouvait la trouver dans une linguistique qui posait en postulat que ses observations portaient sur le substrat formel de la langue, c'est-à-dire, sur ce qui, dans la langue, était non directement lié à l'utilisation ou aux usages qui en sont faits. Or, pour une double raison, c'est cette « mise en service » du langage, plus que sa simple orientation pragmatique, qui nous préoccupe surtout: d'abord parce que les discours auxquels nous étions confrontés avaient pour caractéristique commune qu'ils accompagnaient ou élucidaient certaines manipulations spécifiques en contexte, et ensuite parce que nous avions fait le projet, également très pragmatique, de transmettre par des moyens appropriés ces discours à des communautés de spécialistes souhaitant les acquérir. L'observation des textes, comportements, montages divers entre types de discours, outils et opérations technologiques, les interférences multiples entre productions linguistiques et événements référentiels allaient devenir notre champ de découverte. Les corpus étaient notre seul moyen d'accéder à ces phénomènes à la fois extensifs, enchevêtrés et éminemment labiles.

3 Les corpus, souvent interrogés, encore plus souvent pris pour preuve, n'ont pas pour autant été toujours correctement utilisés. Si l'on en juge sur la foi des résultats produits dans la recherche récente, tant sur le plan international que dans le cadre restreint de notre communauté de recherche, on découvre que les conclusions sur lesquelles on débouche sont encore trop peu abouties, qu'elles sont parfois incomplètes et contradictoires, le plus souvent aussi, difficilement reproductibles sur d'autres corpus. J'en veux pour preuve les études récentes, nombreuses, sur les précautions oratoires ou modalisations phrastiques ("hedges») qui émaillent le discours scientifique. Les plus invraisemblables identifications, comptages, et interprétations, à partir des corpus les plus divers, ont été proposés sans que ne se dégage un consensus de recherche fiable. Force est donc de constater que de graves erreurs théoriques et méthodologiques se sont produites. Ces erreurs me semblent liées à une utilisation massive mais non contrôlée des corpus, et surtout, des instruments qui permettent d'y accéder. Il est dans le propos de cette introduction de recentrer l'usage de cet outil indispensable à notre recherche à la lumière des développements récents en la matière.

4 L'exemple des «linguistes de corpus» britanniques, si souvent présents dans nos colloques de recherche, aurait dû nous guider. Leur démarche et leurs avancées sont passées presque inaperçues. Ils étaient, dès les années 80 , aidés par un certain nombre de facteurs favorables: l'opportune complémentarité entre des spécialistes de disciplines connexes, essentiellement linguistes et informaticiens, la mise en route de très ambitieux projets terminologiques (Cobuild par exemple) et un empirisme conceptuel très largement centré sur les thèses sociolinguistiques de linguistes incontestables comme J. R. Firth et M. A K. Halliday. Autant de facteurs qui auraient pu atténuer l'impact de cette recherche sur le plan scientifique et qui, tout au contraire, ont contribué à lui donner un 
développement maximal. Il faut se rendre à l'évidence, les acquis de la linguistique britannique contemporaine sont immenses (cf. ma bibliographie très sélective).

Est-il possible de "prendre le train en marche » et de prendre appui sur ces acquis de recherche ? Il apparaît d'ores et déjà qu'un certain nombre de principes, qui n'avaient pas, d'ailleurs échappé à la linguistique traditionnelle, vont désormais prendre force de loi : les régularités grammaticales devront être énoncées en regard des contraintes lexicales; la distinction autrefois sacro-sainte entre ces deux catégories et la notion même de règle seront à revoir ; il faudra aussi accepter qu'entre modèle linguistique et usage de la langue, il n'y ait pas une simple relation d'induction mais une relation dialectique.

Si l'on accepte ces principes, est-il possible dans le cadre de la langue spécifique d'avoir recours aux immenses corpus dont ils sont issus? Si la tâche semble profitable du point de vue de la langue générale (cf. les travaux incisifs et perspicaces dans ce numéro de J. Rézeau et les références qu'il donne concernant les corpus commercialisés), il ne me semble pas possible d'y avoir recours dans le cadre des études qui nous occupent. Il s'agit, en effet, de corpus généralistes qui visent à cerner un état de la langue à un moment donné de son histoire. Pour acquérir cette représentativité, ils doivent être les plus étendus possible et doivent contenir tous les types de discours caractéristiques d'une culture donnée. Cette ambition de faire un « état des lieux » de la langue anglaise, peutêtre contestable en soi, a du moins le mérite d'être à l'origine du renouveau des études lexicographiques, lexicologiques et linguistiques en Grande-Bretagne. Pour ce qui nous concerne, plus précisément, les principes mêmes qui ont présidé à la constitution de ces corpus suffisent à les disqualifier dans notre pratique. Ce sont les spécificités du discours scientifique et technique qui nous intéressent. Même s'il est peut-être scientifiquement spécieux et circulaire de prétendre que ces spécificités existent en se consacrant uniquement aux textes dans lesquels elles sont censées se manifester, je ne vois pas d'autre façon de les caractériser sinon essayer de les rassembler en corpus homogènes. Précisément, le recours comparatif aux grands corpus généralistes devrait permettre de trancher une fois pour toutes la question cruciale de la spécificité.

7 Dans ces conditions les grands corpus généralistes ne semblent pas être directement utilisables. Mais, d'ailleurs, faut-il nécessairement avoir recours à de "grandes machines » informatisées sur le modèle des grands corpus britanniques ou américains ? Il est possible, et même, de mon point de vue, souhaitable, de travailler sur des corpus réduits, des prélèvements ou échantillonnages en quelque sorte. Il est important, en effet, de définir précisément où se trouve la spécificité que l'on veut observer et de quelle façon on entend l'aborder. Et, pour ce faire, il faut éviter de faire confiance à la seule intuition, à des étiquettes aussi vagues que " scientifique », «technique » ou « spécialisé », il faudra aussi résister aux suggestions de spécialistes, ne pas se fier non plus à l'état des lieux que l'on peut faire dans un domaine donné. La spécificité en corpus devra être établie avec la plus grande minutie sauf à affaiblir les observations que l'on se propose de faire et à retomber dans les pièges du discours linguistique généraliste.

8 Cette définition des corpus en langue spécifique pourra s'organiser autour de différents principes : la fonction des textes, indépendamment de leurs contenus ; la description d'un même type d'opérations (mise en place d'un logiciel, par exemple) ou d'un ensemble cohérent de concepts (la photosynthèse) ; le discours d'une communauté scientifique ou technique bien paramétrée (cf. le corpus présenté dans ce numéro par Gledhill).

\section{L'objectif est d'éviter :}



la géomorphologie, même si ces deux domaines peuvent être également considérés comme scientifiques, sinon peut-être une certaine similitude de démarche au niveau de l'exposé scientifique (voir le critère « fonction » ci-dessus) ;

- la confusion des finalités discursives : un texte didactique a, certes, des points communs avec un article scientifique traitant du même sujet, mais leur structure discursive n'est en aucun point comparable dans la mesure ou l'un - le texte didactique - vise à communiquer par des moyens qui lui sont propres une information déjà établie sans la remettre en cause, et l'autre - l'article de recherche - cherche à confirmer/infirmer un paradigme scientifique ;

11 - le mélange des types de spécification : même si les discours sont parfois directement adjacents, comme l'étude des mécanismes d'une pathologie donnée, les techniques thérapeutiques pour la combattre et la pharmacologie s'y rapportant, ces textes ne présentent au niveau du discours que des contiguïtés trompeuses. On parle des mêmes choses mais dans une optique discursive fondamentalement différente. Le premier type de discours propose une explication des phénomènes, le second décrit une pratique et le dernier se contente d'établir des classifications. Rien de cohérent ne peut sortir d'un corpus hétérogène de ce type sinon des régularités vagues et non généralisables.

12 - À cela, il faut ajouter les disparités liées aux différents niveaux de technicité que l'on peut rencontrer dans les textes. Rien ne rapproche, sinon un référent commun qu'il faudra isoler et qui risque d'être considérablement déformé au point d'être parfois méconnaissable, la description pharmaceutique d'un médicament et sa présentation dans les notices à l'usage des patients. Les mettre sur le même plan en corpus revient à assimiler la chrysalide à l'insecte parfait ou, pour mieux dire, à confondre la carpe et le lapin. Retrouver des similitudes est un autre problème mais qui ne passe pas nécessairement par l'étude des corpus.

13 L'établissement de la cohérence interne du corpus est une étape incontournable dans notre recherche. Il faut ensuite établir sa définition. On remarque encore trop souvent une confusion entre banques de données et corpus. La banque de données en tant qu'archive de textes rassemble sur support électronique (ou autres) un ensemble (souvent évolutif, cf. l'article de Chukwu) de textes non différenciés; un corpus est toujours une sélection et une construction issue d'un projet de recherche. Cette construction assigne aux banques de données brutes trois types de spécifications :

- une typologie des textes, par exemple les notices d'installation d'appareils informatiques ;

- une utilisation, par exemple étudier le rapport texte/référent machine ;

- un certain degré d' « enrichissement » (voir ci-dessous).

L'étape suivante est de déterminer la taille du corpus (cf. ici même les analyses et les évaluations de $\mathrm{P}$. Bachschmidt). Je ne crois pas à l'effet de masse en langue spécifique. Dès l'instant où c'est la spécificité qui est en question, et non la représentativité comme dans les corpus généralistes, l'étoffement du corpus s'arrête dès que cette spécificité cesse de se manifester de façon significative. Ce point est atteint dès l'instant où l'ajout de nouveaux textes cesse d'introduire de nouveaux termes spécifiques (voir plus bas pour la détermination de ces termes), de changer la pondération entre termes spécifiques et non spécifiques, et où les collocations observées et leurs valeurs respectives ne sont plus modifiées par l'adjonction de nouveaux textes. 
15 Si l'on passe sur les phases plus fastidieuses mais indispensables de saisie/scannage et relectures, viennent ensuite toutes les opérations indispensables qui consistent à " enrichir » les corpus. Il y a d'une part les enrichissements informatiques qui touchent au codage des textes à intégrer au corpus. Ces textes, en effet, doivent être identifiables en tant que tels (au niveau de leur en-tête bibliographique) et dans leur structure (dans leur composition en parties, sous-parties et paragraphes et dans le statut des différentes parties du texte, ex. titres/faux-titres) de façon à ce que toute référence au corpus puisse être immédiatement localisée. Le codage (ou « marquage ») facilite la consultation par les utilisateurs, l'échangeabilité et le partage des données du corpus et son éventuel transfert. Cette étape, à laquelle participera le linguiste, est essentiellement du ressort de l'informaticien. Divers systèmes de codage sont disponibles mais il semble que les systèmes les plus généralement adoptés sont SGML ou TEI.

16 L'autre forme d'enrichissement est de nature linguistique. Elle consiste à affecter à chacun des mots du corpus une étiquette décrivant sa catégorie morpho-syntaxique et éventuellement son genre et son nombre, s'il s'agit d'un substantif français, par exemple. Des logiciels spécifiques sont affectés à cette tâche, les parsers ou taggers, étiqueteurs en français. Il est coutume d'y associer des lemmatiseurs qui rassemblent autour du même lemme, souche lexicale, des mots qui pourraient être dispersés en fonction de leurs variations orthographiques et grammaticales (AMI(e/s) : amical, amitié, amiable). Comme J. Sinclair, et bien que le point soit discutable, je ne préconise pas, au moins dans un premier temps de recherche, des corpus étiquetés et lemmatisés. Il me paraît évident que lorsque ces opérations ont été pratiquées, les phénomènes auront déjà été filtrés en fonction d'une certaine optique d'observation et que, dans une plus ou moins grande mesure, c'est cette optique qui va de nouveau réapparaître (ou transparaître) dans les conclusions de recherche. Je suis pour le contact le plus immédiat avec le donné brut d'observation qui permettra de percevoir un maximum de phénomènes, qui me mettra en présence des aberrations éventuellement présentes en corpus au lieu de les éliminer, qui me donnera l'occasion de forger mes concepts descriptifs en fonction des réalités sans préalable conceptuel. Même s'il y a des risques évidents associés à cette approche, elle me paraît seule de nature à faire progresser valablement la recherche: comme le dit justement J. Sinclair :

If [...] the objective is to observe and record behaviour and make generalisations based on observations, a means of recording structures must be devised which depends as little as posible on theory. The more superficial the better (Sinclair $1987: 107)$.

17 Ainsi, il n'est pas sûr que dans un corpus étiqueté la différence évidente en fréquence entre la fonction de « about » prépositionnel à valeur localisatrice (pratiquement absente de mon corpus <corpus-témoin, en quelque sorte, choisi pour l'exemplification de cette préface, constitué de 10000 mots représentant quatre articles de synthèse sur la structure des immunoglobulines>) soit dissociée de la valeur notionnelle («About the subject of... ») qui, elle, entre dans une distribution pratiquement égale avec "about» approximateur : ex. «About 100 amino-acid residues ». L'important est comment ces valeurs se positionnent les unes par rapport aux autres, en quels contextes elles apparaissent, et pourquoi certaines autres valeurs se trouvent être occultées.

18 Toute préparation du corpus - pour certains indispensable - est de mon point de vue une façon d'éviter - ou risque de fausser - la décision la plus importante qui doit précéder toute interrogation des corpus. Il ne peut y avoir de recherche sans que soit formulée, au 
préalable, une hypothèse de recherche qui va guider non seulement la constitution du corpus mais sa consultation. Pas de recherche sans intention de recherche et point d'intention valable sans confirmation au contact des réalités. Le corpus, quelle que soit son origine ou sa taille, ne peut dispenser le chercheur d'un questionnement. C'est ce questionnement seul qui donne du sens à sa recherche. Paradoxalement aussi, il est important de ne pas se laisser enfermer dans le cadre rigide d'une hypothèse de départ. Il s'agit de maintenir du mieux possible une dialectique de recherche entre le posé et le donné, entre l'hypothèse et sa vérification. L'équilibre, s'il n'est pas facile à maintenir, est néanmoins capital.

19 Il est même possible que le jeu de cette dialectique aille jusqu'à remettre en cause la composition du corpus. Le corpus n'est pas une réalité intangible - un donné brut paré de toutes les grâces de l'authenticité -, il est une réalité construite, évolutive et interactive résultant du travail de l'observateur à la recherche de son objet et des concepts descriptifs s'y rapportant.

Les dernières consignes pour le bon usage des corpus, toujours du point de vue de mon expérience, s'entend, concernent les outils dont on va se servir pour aborder, traiter et explorer ces étendues démesurées de textes : il faut savoir «faire parler » les corpus. Ces outils doivent être les plus simples possible et faire le moins obstacle au regard de l'observateur. Ils doivent s'adapter aux besoins du chercheur et non le contraire. Ils doivent être parfaitement accordés aux caractéristiques du corpus. Ils produisent trois types d'information: des comptages utiles pour établir avec précision quel est le "vocabulaire» spécifique d'un corpus donné, isoler les mots-clés, indiquer leur fréquence, leur densité; des statistiques utiles pour établir, par exemple des rapports entre mots sémantiques et mots outils, pour établir des données comparatives entre corpus, tout particulièrement entre corpus spécifiques et corpus généralistes, et, finalement, des collocations (grâce à des "concordanciers ») permettant d'établir des contextes d'occurrences (à gauche et/ou à droite du terme choisi, avec alignement alphabétique sur le contexte à droite ou à gauche, avec un mot vedette lemmatisé, etc.) ou des réseaux de co-occurrence entre plusieurs termes qui ont tendance à apparaître ensemble (ou qui au, contraire, n'apparaissent jamais en collocation). Ainsi, par exemple, dans mon corpus-test, il est clair que dans l'exposé des conclusions de recherche, n'apparaissaient jamais des termes aussi fréquents et aussi prévisibles que "prove», "imply», «mean», "signify» ou "evince», mais que les prédicats les plus employés étaient "indicate» (pour des résultats avérés) et «suggest» (pour des résultats plus nuancés) ; «show », en revanche, était utilisé, uniquement sous des formes fléchies telles que « showing »/« shown», pour présenter non pas des résultats mais des illustrations non linguistiques. Ces résultats sont, bien sûr, à confirmer mais ils constituent le premier temps du mouvement dialectique dont je parlais plus haut, la première étape dans la mise en évidence de régularités, certes peu éclatantes à ce stade, mais solides et reproductibles. Ce type d'observations statiques et purement descriptives devrait bientôt conduire à des régularités dynamiques qui rendraient compte de la cohérence et de la progression textuelles.

21 Ainsi, dans mon corpus, si l'on observe la fonction rhétorique de "although ", il est frappant de constater que la position initiale, correspondant à une proposition complète, décrit une objection que l'on n'entend pas réfuter et dont il faut tenir compte, alors que la position incidente, entre deux virgules, est souvent associée à une proposition tronquée (" although to a lesser degree ») et se rapporte à l'expression d'une nuance importante mais 
qui n'invalide pas les résultats rapportés. Dans un cas le discours se complexifie; dans l'autre, il se développe presque sans interruption. De telles observations, si elles se multipliaient, permettraient de voir comment la communication scientifique progresse à la fois comme reconfirmation d'un texte paradigmatique préexistant et à la fois comme remise en cause de ce même texte.

Les avantages de la recherche sur corpus sont évidents. Les désavantages que nous allons énoncer ne les invalident aucunement. Ils doivent entrer dans la nécessaire relativisation des résultats à laquelle doit procéder toute démarche scientifique véritablement rigoureuse. Ces désavantages sont de deux ordres : je passerai très vite, même s'ils sont extrêmement chronophages, sur les désavantages techniques concernant la constitution physique des corpus essentiellement dus à la « cécité » de la machine, pour m'appesantir sur les désavantages méthodologiques, et sur la façon de les neutraliser, au moins en partie.

Les outils d'exploration des corpus travaillent dans le quantitatif. Ils n'ont rien à nous apprendre sur les phénomènes uniques ou rares, ni même sur la densité variable des phénomènes qu'ils nous restituent sous forme de bilans chiffrés.

Les outils statistiques privilégient la surface linguistique des textes et écrasent leur spécificité. Ils nous disent bien en quoi ils se ressemblent, mais ne peuvent rendre compte de leur individuelle physionomie.

Les concordanceurs privilégient les phénomènes de proximité et de surface aux dépens des relations plus distendues ou qui opèrent à divers niveaux de profondeur. Ils mettent en valeur les récurrences remarquables tout en minorant les phénomènes isolés ou atypiques.

Tous ces outils tendent à réduire l'emprise humaine de l'énonciateur/scripteur sur son texte qui devient une simple suite de signes alignés "au kilomètre». Or, il faudra conserver sans arrêt à l'esprit que ces textes «allongés » comme des anchois dans leur boîte, sont le produit d'une stratégie argumentative complexe et que, de façon plus cruciale encore, la construction de leur sens dépend du parcours de lecture d'un lecteur qui ne saurait être assimilé au décodage d'un ordinateur aussi performant soit il.

Plus généralement, le texte systématiquement «décortiqué » tend à perdre ses déterminations socio-culturelles, son habillage discursif et les marques de sa destination, pour n'apparaître que comme ce qu'il ne saurait être, c'est-à-dire comme un simple donné physique.

Tous ces désavantages sont réels mais ils ne sont pas rédhibitoires. Ils rendent encore plus impérative l'obligation de formuler des hypothèses de recherche solides, non seulement sur la spécificité, mais aussi sur la discursivité. Ces hypothèses guideront les procédures d'exploration permettront d'en relativiser les résultats et de les cadrer dans une théorie d'ensemble du discours spécifique.

Les corpus sont des outils indispensables pour la recherche aussi bien en tant que terrains d'expérimentation, pour tester exhaustivement des hypothèses, qu'en tant que terrain de découverte pour en susciter de nouvelles. La boucle épistémologique de la recherche est, on le voit, bouclée rendant les résultats à la fois vérifiables et falsifiables. J'en veux pour preuve les différents articles contenus dans cette livraison de ASp consacrée aux corpus. 


\section{BIBLIOGRAPHIE}

« Dossier corpus : de leur constitution à leur exploitation ». 1996. Revue Française de Linguistique Appliquée 1-2.

Gledhill, C. 2000. Collocations in Science Writing. Language in Performance Series 22. Tübingen : Gunter Narr Verlag.

Hoey, M. 1991. Patterns of Lexis in Text. Oxford : Oxford University Press.

Sinclair, J. 1991. Corpus, Concordance, Collocation. Oxford : Oxford University Press.

\section{NOTES}

1. Notamment celui, tout récent, de l'AFLA sur "Construction et utilisation des grands corpus » (Paris, 24-27 sept. 1997) qui a réuni un grand nombre de ceux, de tous horizons, qui, en France et à l'étranger, intègrent des corpus à leur recherche. Voir également le très riche numéro spécial que la Revue Française de Linguistique Appliquée (1996) consacre aux corpus.

\section{RÉSUMÉS}

Cette étude introductive définit l'incidence et l'importance des corpus sur la recherche en langue de spécialité. Sont abordés à cette occasion les divers outils d'enrichissement et d'exploitation des corpus. Une évaluation de l'utilité des corpus en langue de spécialité est proposée en conclusion.

This article is an introduction to the use of corpora in the research on special languages. The definition and specification of specialised corpora is envisaged. A general assessment as to the appropriateness of corpora in ESP research is tentatively proposed.

INDEX

Keywords : corpora in corpus-based research, corpus, corpus linguistics

Mots-clés : corpus, linguistique de corpus, recherche sur corpus

\section{AUTEUR}

JACKY MARTIN 